\title{
Intravitreal Pegaptanib in Ischemic CRVO Refractory Exudative Macular Detachment
}

\author{
Giovanni Cillino and Salvatore Cillino*
}

Department of Experimental Biomedicine and Clinical Neuroscience, Ophthalmology Section, University of Palermo, via Liborio Giuffrè, 13,90127 - Palermo, Italy

\begin{abstract}
Recent literature data reported evidence of the visual and/or anatomical benefits of all clinically available anti-VEGF drugs for the treatment of macular edema (ME) following Central Retinal Vein Occlusion (CRVO), up to 1-year followup. There are no randomized clinical trial data on anti-VEGF agents in ischemic CRVO-ME and the use of anti-VEGF agents to treat this condition.
\end{abstract}

A 73-year old caucasian man, with a medical history of diabetes and systemic hypertension and an ophthalmic history of ischemic CRVO with cystoid macular edema was referred to our division on January 2011. The ophthalmic examination showed in right eye a visual acuity of 20/2000, a C2N1 cataract and an exudative macular detachment. On February 2011, a 0.5mg ranibizumab intravitreal injection (Lucentis ${ }^{\circledR}$, Novartis, Basel, Switzerland) was carried out in the right eye. 15 and 25 days later the OCT showed no changes. On March 2011, we performed a 0.3mg pegaptanib sodium intravitreal injection (Macugen, Eyetech Pharmaceuticals, Inc. and Pfizer Inc, New York, NY) in the right eye and 15 days later the fluorescein angiography and the OCT examination showed reabsorption of subretinal fluid, complete disappearing of macular detachment and normalization of foveal profile, with a thickness of $240 \mu \mathrm{m}$ and visual acuity of 20/400. Three months later, foveal profile and visual acuity were unchanged.

In our refractory ischemic CRVO-ME patient, pegaptanib sodium showed prompt clinical response. In some cases, even if it is selective for the VEGF $_{165}$ isoform only, pegaptanib sodium could represent a further possibility in such a therapeutic challenge.

Keywords: Exudative macular detachment; Central retinal vein occlusion; Intravitreal pegaptanib

\section{Background}

Recent literature data reported evidence of the visual and/or anatomical benefits of all clinically available anti-VEGF drugs for the treatment of macular edema (ME) following Central Retinal Vein Occlusion (CRVO), up to 1-year follow-up [1-3]. Moreover, both ranibizumab and pegaptanib sodium have been effective in refractory CRVO-ME or BRVO-ME case series of patients who had previous bevacizumab and/or triamcinolone injections [4,5]. There are no randomized clinical trial data on anti-VEGF agents in ischemic subgroup CRVO-ME and the use of anti-VEGF agents to treat this condition therefore remains anedoctal.

\section{Case Presentation}

A 73-year old caucasian man, with a general medical history of diabetes and systemic hypertension and an ophthalmic history of non-ischemic Central Retinal Vein Occlusion (CRVO) with cystoid macular edema (CME) in his right eye in December 2008, was referred to our division on January 2011. The patient had undergone 3 triamcinolon injections in 2009 and a Panretinal Photocoagulation after an ischemic shift. The ophthalmic examination showed in this eye a visual acuity of 20/2000, a C2N1 cataract (based on Lens Opacities Classification System III) and an exudative macular detachment. The left eye was normal with a visual acuity of 20/20. We performed fluorescein angiography and Optical Coherence Tomography (OCT3 Stratus, Carl Zeiss, Dublin, CA), the latter showing a foveal thickness of $1718 \mu \mathrm{m}$, measured by caliper (Figures 1 and 2). On 02/09/2011, once obtained the informed consent, a $0.5 \mathrm{mg}$ ranibizumab intravitreal injection (Lucentis ${ }^{\circ}$, Novartis, Basel, Switzerland) was carried out in the right eye. 15 and 25 days later, we repeated the OCT, that showed no changes; the visual acuity was unchanged as well. Due to the

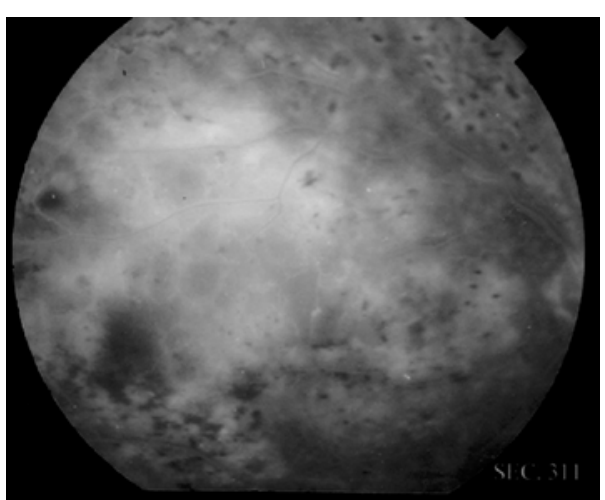

Figure 1: Fluorescein angiography at baseline.

patient's demands, and on the basis of the usually rapid improvement, within 7 days, in visual acuity and foveal thickness in the CRUISE study (ranibizumab), and of the small series from Udaondo et al. [3,5] on

*Corresponding author: Salvatore Cillino, Department of Experimental Biomedicine and Clinical Neuroscience, Ophthalmology Section, University of Palermo, via Liborio Giuffrè, 13. 90127 - Palermo, Italy, Tel: +39 91 6553929; Fax: +39 91 6261438; E-mail: salvatore.cillino@unipa.it

Received December 14, 2011; Accepted January 04, 2012; Published January 10, 2012

Citation: Cillino G, Cillino S (2012) Intravitreal Pegaptanib in Ischemic CRVO Refractory Exudative Macular Detachment. J Clinic Experiment Ophthalmol 3:202. doi:10.4172/2155-9570.1000202

Copyright: (c) 2012 Cillino G, et al. This is an open-access article distributed under the terms of the Creative Commons Attribution License, which permits unrestricted use, distribution, and reproduction in any medium, provided the original author and source are credited. 
Citation: Cillino G, Cillino S (2012) Intravitreal Pegaptanib in Ischemic CRVO Refractory Exudative Macular Detachment. J Clinic Experiment Ophthalmol 3:202. doi:10.4172/2155-9570.1000202

Page 2 of 2

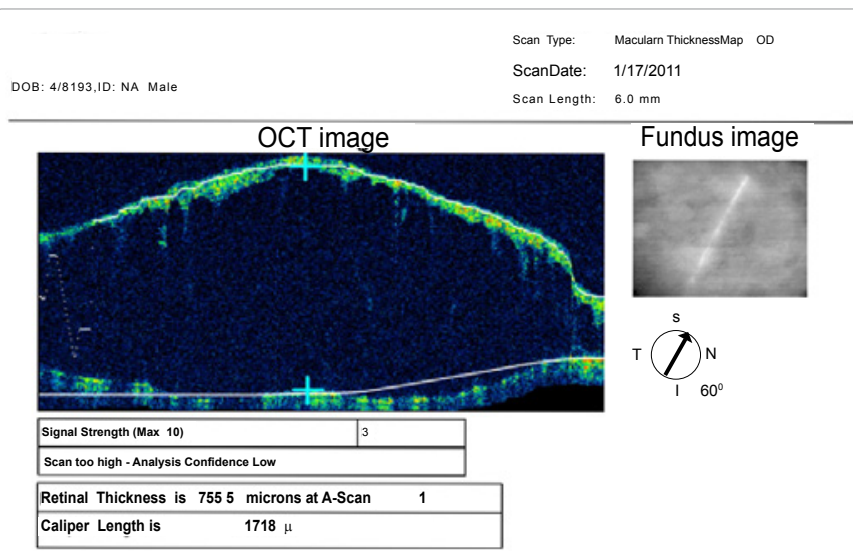

Figure 2: Optical Coherence Tomography (OCT3 Stratus, Carl Zeiss, Dublin, $\mathrm{CA})$ at baseline.

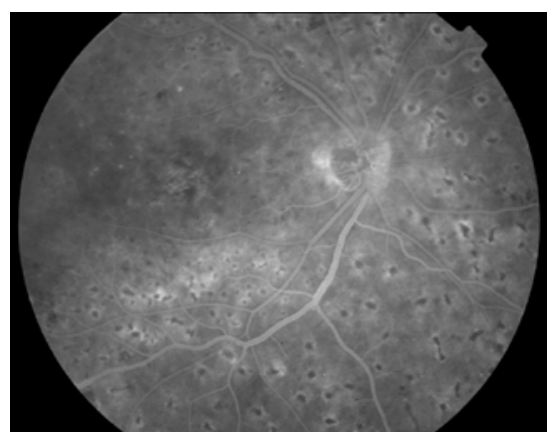

Figure 3: Fluorescein angiography at 15 days follow-up.

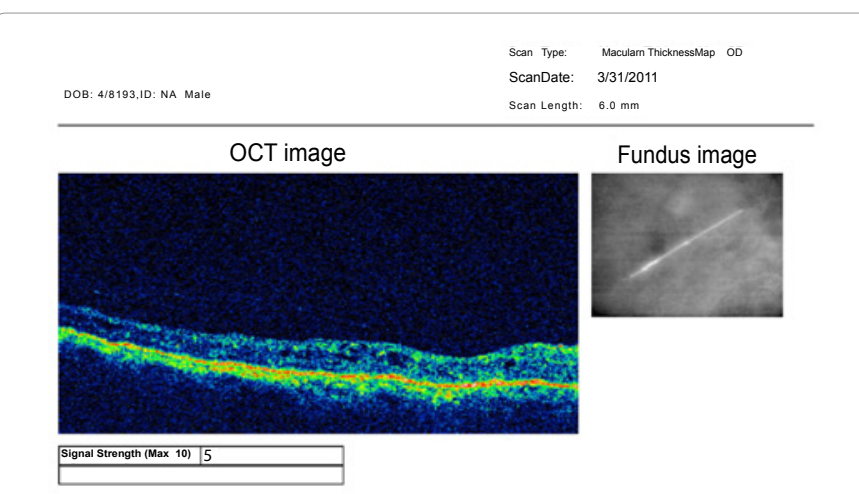

Figure 4: Optical Coherence Tomography (OCT3 Stratus, Carl Zeiss, Dublin, CA) at 15 days follow-up. pegaptanib efficacy, on 03/09/2011, after the written informed consent, we switched to a $0.3 \mathrm{mg}$ pegaptanib sodium intravitreal injection (Macugen, Eyetech Pharmaceuticals, Inc. and Pfizer Inc, New York, $\mathrm{NY}$ ) in the right eye and 15 days later the fluorescein angiography and the OCT examination showed reabsorption of subretinal fluid, complete disappearing of macular detachment and normalization of foveal profile, with a foveal thickness of $240 \mu \mathrm{m}$ and visual acuity of 20/400 (Figures 3 and 4). Three months later, foveal profile and visual acuity were unchanged.

\section{Conclusion}

It is difficult to individuate if the lack of efficacy of ranibizumab in this case is due to the block of the neuroprotectant isoforms of VEGF necessary for the adaptative response of retinal neurons to ischemic injury or if another "trigger" is involved with the pegaptanib response.

Anyway, the observation that, in our refractory ischemic CRVOME patient, pegaptanib sodium showed prompt clinical response, may suggest that at least in some cases this drug could represent a further possibility in such a therapeutic challenge.

Moreover, even if today dexamethasone intravitreal implant has a specific indication for the treatment of macular edema following CRVO, anti-VEGF therapy could well maintain a role due to minimal collateral effects, at least for glaucoma patients or in combination treatments.

\section{Competing Interests}

The authors declare that they have no competing interests.

\section{Authors' Contribution}

GC and SC participated in conception and study design. SC critically appraised the manuscript. All Authors drafted and approved the final manuscript.

\section{References}

1. Wroblewski JJ, Wells JA 3rd, Adamis AP, Buggage RR, Cunningham ET Jr, et al. (2009) Pegaptanib sodium for macular edema secondary to central retina vein occlusion. Arch Ophthalmol 127: 374-80.

2. Braithwaite T, Nanji AA, Greenberg PB (2010) Anti-vascular endothelial growth factor for macular edema secondary to central retinal vein occlusion. Cochrane Database Syst Rev Oct 6: CD007325.

3. Brown DM, Campochiaro PA, Singh RP, Li Z, Gray S, et al. (2010) Ranibizumab for macular edema following. central retinal vein occlusion. Six-month primary end point results of a Phase III study. Ophthalmology 117: 1124-1133.

4. Spaide RF, Chang LK, Klancnik JM, Yannuzzi LA Sorenson J, et al. (2009) Prospective study of intravitreal ranibizumab as a treatment for decreased visual acuity secondary to central retinal vein occlusion. Am J Ophthalmol 147 298-306.

5. Udaondo P, Garcia-Delpech S, Salom D, Garcia-Pous M, Diaz-Llopis M (2011) Intravitreal pegaptanib for refractory macular edema secondary to retinal vein occlusion. Clin Ophthalmol 5: 941-944. 